\title{
Urologic Injuries Associated With Repair of Anorectal Malformations in Male Patients
}

\author{
By Andrew R. Hong, Maria Fernanda Acuña, Alberto Peña, Laura Chaves, and George Rodriguez \\ New Hyde Park, New York
}

\begin{abstract}
Background/Purpose: Serious injuries to the urinary tract may occur during the repair of an anorectal malformation, especially in boys. This review of a large series of patients characterizes factors that may either lead to, or prevent, those injuries.
\end{abstract}

Methods: A retrospective review of 1,003 boys with anorectal malformations was performed.

Results: A total of 129 injuries in 1,003 patients were identified. Five hundred seventy-two of the 1,003 patients (group A) underwent definitive repair at the authors' institution. In this group, there were 19 urologic injuries; 1 bladder perforation, 1 divided ureter, 2 divided vas defera, 1 prostatic injury, 7 seminal vesicles were opened and closed, and in 7 cases, the urethra was opened and closed during the repair. Follow-up ranges from 15 years to 1 month and no late sequelae have been observed. The second group (B) consisted of 431 patients who underwent various operations at other institutions. In this group, 110 urologic injuries in 97 patients were noted. These included neurogenic bladder $(\mathrm{n}=$ 27), persistent, recurrent or acquired recto-urethral fistulae $(n=30)$, posterior urethral diverticulae that required reop- eration ( $n=23$ ), urethral injuries leading to stenosis or aquired atresia $(n=19)$, pull-through of major urinary structures $(n=2)$, injured ureter $(n=1)$, opened seminal vesicle $(n=1)$, divided vas defera $(n=4)$, impotence $(n=1)$, and loss of ejaculation ( $n=2$ ). Several significant associations were noted. The most significant was that all 27 patients with neurogenic bladder and all 19 of those in group B with urethral injuries did not undergo a distal colostogram to define the level of the fistula before repair. Posterior urethral diverticulae were seen only in cases of recto-bulbar urethral fistulae repaired via an abdominal-perineal approach.

Conclusions: Significant urologic injuries are associated with the repair of anorectal malformations. The risk of injury is increased in those patients who undergo repair without a distal colostogram.

J Pediatr Surg 37:339-344. Copyright @ 2002 by W.B. Saunders Company.

INDEX WORDS: Anorectal malformation, imperforate anus, urologic injury, rectourethral fistula, urethral stenosis, neurogenic bladder.
$\mathbf{M}$ ORE THAN $80 \%$ of boys born with anorectal malformations have a fistula between the rectum and the urinary tract. ${ }^{1-3}$ Repair of these defects necessarily involves the separation of these 2 systems. This generates a significant risk of injury to important structures, including the urethra, bladder, ureters, vas deferens, seminal vesicles, prostate, and important nerves responsible for urinary control and sexual function.

We were motivated to present this experience after seeing and treating a large number of patients that suffered from significant, and sometimes multiple, urologic inuries after the surgical repair of imperforate anus.

An awareness of this problem has existed for many years, and numerous reports exist that list several different types of urologic injuries that occur during the repair of anorectal malformations in boys. ${ }^{4-27}$ However, with the exception of the problem of neurogenic bladder, these reports do not discuss the mechanism of injury in detail, and recommendations on how to avoid these injuries are not made. In this large retrospective review of essentially 2 patient populations, we attempt to analyze the possible causes that contribute to this morbidity, as well as make recommendations to help pediatric surgeons avoid these injuries in the future.

\section{MATERIALS AND METHODS}

The medical records and the x-ray studies of 1,003 boys born with an anorectal malformation, and treated and followed by the senior author from 1981 until 2000 were reviewed. Age at the time of surgery varied from 1 month to 33 years. Two groups were identified. Group A included 572 boys who underwent primary repair at our institution using a posterior sagittal approach (PSARP). Group B included 431 boys who underwent their primary repair at another institution and who subsequently came to our hospital for further surgical treatment or bowel management. Any event that could lead to permanent sequela or morbidity or that required additional surgery to be repaired were considered urologic complications. In the event of neurogenic bladder, patients with severe sacral anomalies were excluded because those congenital defects could be responsible for a congenital neurogenic bladder. A literature search on the subject was performed using Med-

From the Division of Pediatric Surgery, Schneider Children's Hospital, New Hyde Park, NY.

Presented at the 32nd Annual Meeting of the American Pediatric Surgical Association, Naples, Florida, May 20-23, 2001.

Address reprint requests to Andrew R. Hong, MD, Division of Pediatric Surgery, Schneider Children's Hospital, 269-01 76th Ave, New Hyde Park, NY 11040.

Copyright (C) 2002 by W.B. Saunders Company

0022-3468/02/3703-0005\$35.00/0

doi:10.1053/jpsu.2002.30810 
Table 1. Urologic Injuries and Type of Operation

\begin{tabular}{llccc}
\hline Group & \multicolumn{1}{c}{ Technique } & Cases & Injuries & Percentage \\
\hline A & PSARP & 572 & 19 & 3.3 \\
B & PSARP & 122 & 39 & 32.0 \\
B & Others & 309 & 77 & 24.9 \\
& Abdomino-perineal & 125 & 40 & 32.0 \\
& Anoplasty & 45 & 11 & 24.4 \\
& Sacro-perineal & 33 & 10 & 30.3 \\
& Sacro-abdomino-perineal & 55 & 4 & 7.3 \\
& No information & 39 & 7 & 17.9 \\
& Other techniques & 12 & 5 & 41.7 \\
\hline
\end{tabular}

line, and relevant papers were reviewed. Institutional Review Board approval was not requested for this retrospective review.

\section{RESULTS}

A total of 129 injuries in 116 patients were found. In group $A$, there were 19 injuries in 572 patients (3.3\%.) In group B, 431 patients sustained a total of 110 injuries $(25.5 \%$.)

The type of surgical approach was correlated to the incidence of injury (Table 1). In group A, all 572 patients underwent PSARP, for an injury rate of $3.3 \%$. In group B, 122 patients underwent PSARP, and there were 39 injuries $(32 \%)$. Three hundred nine patients underwent repair utilizing other techniques, and there were 77 injuries $(25 \%$.)

Six major types of injuries were identified, and they are listed, along with their incidence, in Table 2. There were 26 urethral injuries, 7 in group A and 19 in group B. Posterior urethral diverticulae occurred in 23 patients, all in group B. Thirty patients in group B had a recurrent, persistent or acquired rectourethral fistula. Bladder injuries included 27 cases of neurogenic bladder and 1 bladder pull-through, all in group B, and 1 bladder injury in group A. A total of 18 injuries to the reproductive tract were found, 10 in group A and 8 in group B. Finally, there were 3 ureteral injuries, 1 in group $A$ and 2 in group B.

An attempt was made to correlate the type of malformation and the presence of an injury. However, we found that it was impossible to classify the patients in group B, because in many cases only vague descriptions of the anatomy were available. Table 3 lists the type of malformation and type of injury seen in the 19 patients in group A.

Table 2. Type of Urologic Injuries

\begin{tabular}{lccc}
\hline & Group A & Group B & Total \\
\hline Urethral injuries & 7 & 19 & 26 \\
Recto-urinary fistula & 0 & 30 & 30 \\
Posterior urethral diverticulae & 0 & 23 & 23 \\
Bladder & 1 & 28 & 29 \\
Genitalia & 10 & 8 & 18 \\
Ureteral & 1 & 2 & 3 \\
Total & 19 & 110 & 129 \\
\hline
\end{tabular}

Table 3. Malformations and Injuries-Group A

\begin{tabular}{lccccc}
\hline \multicolumn{1}{c}{ Injury Type } & $\begin{array}{c}\text { Perineal } \\
\text { Fistula }\end{array}$ & $\begin{array}{c}\text { Bulbar- } \\
\text { Urethral }\end{array}$ & Prostatic & $\begin{array}{c}\text { Bladder } \\
\text { Neck }\end{array}$ & Total \\
\hline Urethral & 0 & 3 & 4 & 0 & 7 \\
Recto-urinary & 0 & 0 & 0 & 0 & 0 \\
$\begin{array}{l}\text { Postoperative } \\
\text { Urethral }\end{array}$ & & & & & \\
$\quad$ Diverticulum & 0 & 0 & 0 & 0 & 0 \\
Bladder & 0 & 0 & 0 & 1 & 1 \\
Reproductive & 0 & 0 & 8 & 2 & 10 \\
Ureteral & 0 & 0 & 0 & 1 & 1 \\
\hline
\end{tabular}

The failure to perform a preoperative pressure augmented distal colostogram was evaluated. Twelve patients in group A did not undergo a colostogram. Of those, 4 sustained urinary injuries. In group B, most patients either did not undergo a colostogram or the one that was performed was technically deficient. Of the patients with bladder injuries, all 29 did not undergo a distal colostogram, and none of the 19 patients in group B with urethral injuries had a preoperative colostogram either.

Several of the injuries were examined in greater detail. Table 4 shows the frequency of neurogenic bladder (NB) associated with each type of procedure. All 27 of these cases occurred in group B. There were 12 of 122 (9.8\%) in the PSARP group, 9 of $125(7.2 \%)$ in the abdominoperineal group, 1 of $45(2.2 \%)$ in the anoplasty group, 2 of $33(6 \%)$ in the sacroperineal group, and 3 of $12(25 \%)$ in patients operated on by various other techniques. No cases of NB were found consecutive to sacro-abdominoperineal procedures, nor in the 39 patients from whom we did not have information concerning the type of operation that was performed originally.

Twenty-six patients suffered from a urethral injury. The types of injuries encountered are listed in Table 5. Nineteen were significant and required additional surgery, including 12 stenoses and 7 acquired atresias. It is important to note that in 6 of these cases, the injury involved complete transection of the urethra. All these cases occurred in group B. In group A, we found 7 patients in whom the urethra was opened widely enough to expose the Foley catheter. Primary closure was accomplished in all 7 cases, and no long-term sequelae were observed.

Table 4. Neurogenic Bladder-Group B

\begin{tabular}{lccc}
\hline \multicolumn{1}{c}{ Technique } & No. of Operations & No. of NB & Percentage \\
\hline PSARP & 122 & 12 & 9.8 \\
Abdomino-perineal & 125 & 9 & 7.2 \\
Other techniques & 12 & 3 & 25.0 \\
Sacro-perineal & 33 & 2 & 6.0 \\
Anoplasty & 45 & 1 & 2.2 \\
Sacro-abdomino-perineal & 55 & 0 & 0.0 \\
No information & 39 & 0 & 0.0 \\
\hline
\end{tabular}

NOTE. Group B $(\mathrm{n}=431)$. 
Table 5. Type of Urethral Injuries

\begin{tabular}{llccc}
\hline Group & \multicolumn{1}{c}{ Type } & Injuries & Cases & Percentage \\
\hline A & Incidental opening & 7 & 572 & 1.2 \\
B & Stenosis & 12 & 431 & 4.4 \\
B & Acquired atresia & 7 & & \\
Total & & 26 & & \\
\hline
\end{tabular}

Twenty-three of 431 patients $(5.3 \%)$ in group B had posterior urethral diverticulae. This lesion, which occurs when a segment of the rectum is left attached to the urinary tract, was seen exclusively in patients who underwent an abdomino-perineal approach for a rectourethral fistula.

Thirty postoperative recto-urethral fistulae were seen, all in group B (7\%). These lesions exist in 3 forms, persistent, acquired, and recurrent (Table 6). There were 16 persistent fistulae. In all 16 cases, the presence of a recto-urethral fistula was not recognized at the time of the first operation. There were 2 acquired fistulae. In both cases, patients without a congenital recto-urethral fistula underwent an anoplasty at their first operation, and subsequently a recto-urethral fistula developed. In both cases, the operation was performed without a Foley catheter in place. There were 12 recurrent fistulae, which occurred in spite of a correct preoperative diagnosis and repair of the fistula at the time of the first operation.

The reproductive injuries that occurred are displayed in Table 7. In group A, 7 patients were noted to have an opened seminal vesicle at the time of their primary repair. This event was recognized in 1 patient in group B. Immediate repair was accomplished in all cases. It is too early in the course of these patients' lives to know the long-term consequences of this event. Six patients sustained division of a vas deferens, 2 in group A and 4 in group B. In addition, in group B, there is 1 patient who is impotent, and 2 are unable to ejaculate. There are no data on fertility.

\section{DISCUSSION}

We have struggled with the best way to present these data. We are well aware of the limitations of a retrospective review of this kind. Furthermore, we are acutely cognizant of the fact that the population in group B is a select population, and is highly unlikely to represent the general experience at other institutions. We certainly do not mean to imply that the general rate of urologic injury is so high.

However, we believe that the number of urologic injuries seen in group B is significant, especially in light of the fact that the majority were most likely preventable. We also believe, in spite of the limitations of the study, the data still can be used to identify the most common causes that contribute to these complications. Finally, based on our experience with the patients in group A, we feel justified in making recommendations that may help pediatric surgeons avoid these complications in the future.

An overview of the results allows us to detect certain patterns. For instance, of all the different operations done at other institutions, the posterior sagittal approach provoked one of the highest incidences of urologic injuries. This is rather distressing, given the authors' well known point of view. We suspect, but cannot prove, that it in part is caused by the selection bias of the patients in group B. Group B patients most likely represent a subset of patients in whom a complication did occur, and then came to our institution for further care.

Another trend that became obvious is that a posterior urethral diverticulum occurs almost exclusively when patients born with a recto-bulbar urethral fistula undergo an abdomino-perineal operation, and a segment of rectum is left attached to the urinary tract. We also found that persistent recto-urinary fistulae occurred when the surgeon failed to recognize the presence of a congenital recto-urethral fistula and attempted to repair the lesion with an anoplasty. Finally, the performance of a preoperative distal colostogram to show the level of the rectourinary fistula appears to have a preventative effect when it comes to urologic injuries.

\section{Risk of Operative Technique}

We found that most of the injuries identified in our study were at least mentioned in the literature we reviewed. ${ }^{4-26}$ However, most did not go into the same type of detail as in the current study. For instance, we felt that it would be useful to try to determine the risk of injury for each type of operative technique used to repair the anorectal malformation (Table 1). Most of the reports did not attempt to compare different surgical techniques. Some investigators have attempted to correlate the surgical approach with the type of injury. Tank et al, ${ }^{11}$ in 1972, reviewed 100 cases of anorectal malformations repaired via an abdomino-perineal approach. He found 2 urethral strictures, 1 persistent fistula, and a $10 \%$ incidence of postoperative neurogenic bladder. They conclude that those injuries were produced only in patients that underwent repair as newborns without a colostomy. They proposed that the repair be delayed until after the patient reached the weight of $25 \mathrm{lbs}$. Nakayama et $\mathrm{al}^{19}$ in 1986 reported complications that occurred only consecutive to posterior sagittal operations, including a recur-

Table 6. Postoperative Recto-Urethral Fistulae-Group B

\begin{tabular}{lrr} 
Table 6. Postoperative Recto-Urethral Fistulae-Group B \\
\hline Persistent fistula & 16 & $3.7 \%$ \\
Recurrent fistula & 12 & $2.7 \%$ \\
Acquired fistula & 2 & $0.4 \%$ \\
Total & 30 & $6.9 \%$ \\
\hline
\end{tabular}

NOTE. Group B $(n=431)$. 
Table 7. Reproductive Injuries (1,003 Patients)

\begin{tabular}{|c|c|c|c|c|c|}
\hline & \multicolumn{2}{|c|}{ Group A } & \multicolumn{2}{|c|}{ Group B } & \multirow[b]{2}{*}{ Totals } \\
\hline & PSARP & PSARP & Sacro-Perineal & Abdomino-Perineal & \\
\hline Divided vas deferens & $2 / 572$ & $3 / 122$ & $1 / 33$ & 0 & 6 \\
\hline No ejaculation & 0 & 1 & 0 & 1 & 2 \\
\hline No erection & 0 & 0 & 0 & 1 & 1 \\
\hline Open seminal vesicle & $7 / 572$ & $1 / 122$ & 0 & 0 & 8 \\
\hline Prostatic opening & $1 / 572$ & 0 & 0 & 0 & 1 \\
\hline
\end{tabular}

rent recto-urethral fistula. McLorie et $\mathrm{al}^{27}$ in 1998 reported 6 cases of serious urologic injuries that occurred during the repair of an anorectal malformation. All of the patients were operated on posterior sagitally. Three patients suffered from partial urethral disruption, and 3 others had complete disruptions of the urethra from the bladder. They concluded that the PSARP may make the appreciation of the anatomy more difficult.

\section{Urethral Injuries}

Urethral injuries have been mentioned by many investigators. ${ }^{4,6,10-13,15-19,26}$ Our own experience includes 7 patients in whom the foley catheter was exposed during the separation of the rectum from the urinary tract. The problem was recognized immediately, and the urethra was repaired without long-term consequences. These problems occurred early in our series, and we have not seen a similar case during the last 13 years. A review of the operative reports of the 19 patients that suffered severe urethral injuries at other institutions found several interesting factors. Ten of the patients originally underwent PSARP. In all 10 cases, no preoperative distal colostogram was performed. Consequently, the posterior sagittal approach was performed without prior knowledge as to the level of the fistula. In this situation, the likelihood of urethral injury clearly is increased. Eight patients suffered urethral injuries during the performance of an abdomino-perineal procedure. One can only speculate as to the cause of the injury. We suspect that it results when excessive traction is applied during the dissection of the rectum, leading to transection or injury to the urethra. Three patients sustained urethral injuries during the repair of a perineal fistula. All 3 underwent anoplasty without a catheter in the urethra. A urethral injury in this type of malformation is extremely unfortunate, because these patients have an excellent prognosis. It is extremely important for the surgeon to keep in mind that, even in this type of defect, the rectum still is intimately attached to the urethra, and a meticulous dissection and separation of the 2 structures is necessary. The presence of a Foley catheter can only aid in the identification of the anatomy.

We believe that urethral injuries are $100 \%$ preventable. Except in cases of recto-perineal fistulas, the repair never should be performed without a preoperative distal colostogram. ${ }^{28}$ We believe that this is the most valuable study in the management of anorectal malformations. A search for the rectum in the operating room without advance knowledge of the location of the fistula, clearly will lead to an increased chance of injury.

\section{Neurogenic Bladder}

Neurogenic bladder after the repair of an anorectal malformation has been mentioned by most of the investigators. ${ }^{9-13,15,16,18,21-25}$ Tank ${ }^{11}$ reported a $10 \%$ incidence of neurogenic bladder. Wiener and Kiessewebber ${ }^{13}$ studied 200 patients and found an $18 \%$ incidence of neurogenic bladder. They believed that only 3 of them were secondary to iatrogenic causes. Ralph et al ${ }^{23}$ reported on 58 patients referred for urology consultation after repair of an anorectal malformation. Thirty-two of those 58 patients $(55 \%)$ suffered from neurogenic bladder! Twenty-nine of them were hyperreflexic, and 3 were atonic. Ralph et $\mathrm{al}^{23}$ are some of the very few investigators who try to clarify the difference between a neurogenic bladder that is congenital and one that is secondary to surgery. They believe that the atonic type of neurogenic bladder could be attributed to surgical injury, whereas the 29 patients with hyperreflexic bladders most likely had some form of upper motor neuron deficiency. In support of this conclusion is that all 29 had significant spinal abnormalities associated with their malformations. Boemers et $\mathrm{al}^{25}$ reported the most comprehensive series of patients, evaluating the degree of damage that resulted from the surgical repair. In their report, a study of 27 cases, urodynamic studies were performed before and after surgery. They concluded that the posterior-sagittal anorectoplasty and its variants do not affect lower urinary tract function unless the surgical techniques are combined with major transabdominal procedures and extensive retrovesical dissection. This conclusion is supported by our findings when group $\mathrm{A}$ is considered alone because no case of NB was seen in this group. However, in group B, 12 of the 122 patients who underwent PSARP had NB (10\%). When we examined these cases to ascertain a possible cause, we found that all $12 \mathrm{did}$ not undergo a preoperative distal colostogram. Our decision to eliminate patients with significant sacral abnormalities 
from consideration is supported by previous work. Boemers et $\mathrm{al}^{25}$ performed urodynamic studies in children with anorectal malformations and found that a normal sacrum correlated with normal lower urinary tract function, and anormal function correlated with partial or severe sacral agenesis.

\section{Postoperative Recto-Urethral Fistulae}

A recto-urethral fistula after an operation for imperforate anus is a well known problem. 5,6,9,11,14-17,19,20 Duffy, ${ }^{5}$ in 1951, published an interesting case of a patient who underwent surgical repair as a child but was left with a persistent fistula, which was discovered 27 years later! Spence 6 reported one case of an acquired rectourethral fistula after an anoplasty in 1954. Williams and Grant, ${ }^{9}$ in 1969 , described a persistent recto-urethral fistula that he repaired by interposing omentum between rectum and urethra. Tank et $\mathrm{al}^{11}$ mentioned one case of a persistent recto-urethral fistula. Carlton et al ${ }^{14}$ described 3 recurrent recto-urethral fistulas in 1973. Finally, Persky et al ${ }^{15}$ also described a case of a persistent urethral fistula in 1974.

We found that postoperative recto-urethral fistulae occur in 3 forms: those that are persistent, those that are acquired, and those that recur. Sixteen patients in group B suffered from a persistent recto-urethral fistula (Table 6). Clearly, this is because the existence of a congenital fistula was not recognized at the time of the initial repair. Twelve patients suffered from a recurrent recto-urethral fistula. In these cases, the congenital fistula was recognized and surgically repaired but subsequently recurred. We speculate that either anastomotic dehiscence or injury to the rectal wall plays a role in this event.

Acquired recto-urethral fistulae occurred when a recto-perineal fistula was repaired, and the urethra was injured during the anoplasty. This happened in 2 cases and was associated with the lack of an intraoperative urethral catheter both times.

\section{Posterior Urethral Diverticulum}

The posterior urethral diverticulum also has been reported in the literature. $8,9,12,13,15-18,20$ The first reports that we were able to find came from Currarino ${ }^{8}$ and Williams and Grant. ${ }^{9}$ Williams and Grant mentioned their concern about the formation of stones inside the piece of rectum attached to the urethra and only operated on those patients when they had a stone.

In our series, there were 23 of 431 patients in group B with this problem and none in group A. As mentioned previously, this problem occurrs when an abdominoperineal approach is used to repair recto-urethral fistulae, and an excessively long segment of rectum is left attached to the urethra. Pediatric surgeons should be aware of this complication, especially if they are faced with an adolescent or adult patient with a history of an abdomino-perineal repair and symptoms of urinary incontinence, infections, or stones. Surgical excision of the diverticulum always is indicated, because we have seen one patient in whom malignant transformation in the diverticulum developed. ${ }^{27}$

\section{Reproductive Tract Injuries}

Eighteen patients suffered from some form of reproductive tract injury (Table 6). Five of them occurred during posterior sagittal operation performed at other Institutions, 10 during PSARP performed by us, one during a sacro-perineal approach, and 2 during abdomino-perineal operations. We found only 1 report, that of Carlton et al, ${ }^{14}$ that discussed these injuries. Their report mentioned 1 case in which a vas deferens was divided. In our series, there were 6 vas injuries: 2 in group A and 4 in group B. In at least 5, including both patients in group A, there was no preoperative distal colostogram, and the vas was injured during a search for a very high rectum. The importance of the distal colostogram has since been recognized and is now routinely utilized in our practice.

In a total of 8 cases, a seminal vesicle was opened at the time of primary repair. In all cases, immediate repair of the injury was performed. No short-term sequelae were observed, but it is too early to comment on the long-term consequences of this event. Seven of these were in group A. It is possible that the incidence of this event in group B is higher, but the complication was not recognized and consequently not reported. It is our experience that the seminal vesicles lie very close to the rectum and that a meticulous dissection at the level of the rectal wall is necessary to avoid injuring adjacent reproductive structures such as seminal vesicles or the prostate.

There is a significant risk of urologic injury during the repair of an anorectal malformation in a male patient. The posterior sagittal approach, if performed without a preoperative distal colostogram, has a significantly higher risk than other surgical approaches. There are 6 major types of injuries seen, many of which can be avoided by the appropriate preoperative workup and intraoperative management. The preoperative pressure augmented distal colostogram appears to be the most important test that can be done to avoid intraoperative urologic complications. We recommend the placement of Foley catheters during the repair of all of these malformations, including perineal fistulas. Pediatric surgeons should suspect a posterior urethral diverticulum in any patient with urinary symptoms and a history of a repair via an abdomino-perineal approach. 


\section{REFERENCES}

1. Peña A: Anorectal malformations. Semin Pediatr Surg 4:35-47, 1995

2. Peña A, Hong AR: Anorectal malformations-The State of the Art. Colon and Rectal Surgery 2:1-19, 1999

3. Stephens FD, Smith ED: Incidence, frequency of types, etiology, in Stephens FD, Smith ED (eds): Ano-rectal Malformations in Children. Chicago, IL, Year Book Medical, pp 160-171, 1971

4. Wallace FT, Colvin EM: Complications of imperforate anus repair. Surgery 24:832-835, 1948

5. Duffy JJ: Imperforate anus and recto-urethral fistula in a twentyseven year old man. JAMA 145:545-548, 1951

6. Spence HM: Anomalies and complications of the urogenital tract associated with congenital imperforate anus. J Urol 71:453-463, 1954

7. Smith ED: Urinary anomalies and complications in imperforate anus and rectum. J Pediatr Surg 3:337-342, 1968

8. Currarino G: Diverticulum of prostatic urethra developing postoperatively from stump of congenital rectourethral fistula. Am J Roentgenol 106:211-215, 1969

9. Williams DI, Grant J: Urological complications of imperforate anus. Br J Urol 41:660-665, 1969

10. Carcassonne M, Monfort G, Isman H: Les problemes urologiques des malformations ano-rectales. Arch Franc Ped 28:723739, 1971

11. Tank ES, Erns CB, Woolson ST, et al: Urinary tract complications of anorectal surgery. Am J Surg 123:118-122, 1972

12. Belman AB, King LR: Urinary tract abnormalities associated with imperforate anus. J Urol 108:823-824, 1972

13. Wiener ES, Kiessewetter WB: Urologic abnormalities associated with imperforate anus. J Pediatr Surg 8:151-157, 1973

14. Carlton CE Jr, Harberg FJ, Fry FM: Urologic complications of imperforate anus. J Urol 109:737-739, 1973

15. Persky L, Tucker A, Izant RJ: Urological complications of correction of imperforate anus. J Urol 111:415-418, 1974

16. Parrot TS: Urologic complications of imperforate anus. Urology 10:407-413, 1977
17. Lebowitz RL, Lamego C: Rectourethral fistula on the urethrogram. Urol Radiol 1:53-59, 1979

18. Parrot TS: Urologic implications of anorectal malformations. Urol Clin North Am 12:136-121, 1985

19. Nakayama DK, Templeton JM Jr, Ziegler MM, et al: Complications of posterior sagittal anorectoplasty. J Pediatr Surg 21:488-492, 1986

20. von Bodmann J, Hecker WC: Two rare complications in operated rectal and anal atresia. Z Kinderchir 43:56-58, 1988

21. Gross GW, Wolfson PJ, Peña A: Augmented-pressure colostogram in imperforate anus with fistula. Radiol 21:560-562, 1991

22. Sheldon C, Cormier M, Crone K, et al: Occult neurovesical dysfunction in children with imperforate anus and its variants. J Pediatr Surg 26:49-54, 1991

23. Ralph DJ, Woodhouse CRJ, Ransley PG: The management of the neuropathic bladder in adolescents with imperforate anus. J Urol 148:366-368, 1992

24. Kakizaki H, Nonomura K, Asano Y, et al: Preexisting neurogenic voiding dysfunction in children with imperforate anus: Problems in management. J Urol 151:1041-1044, 1994

25. Boemers TM, Bax KMA, Rovekamp MH, et al: The effect of posterior sagittal anorectoplasty and its variants on lower urinary tract function in children with anorectal malformations. J Urol 153:191-193, 1995

26. Boemers TML, Beek FJA, van Gool JD, et al: Urologic problems in anorectal malformations. Part I: Utodynamic findings and significance of sacral anomalies. J Pediatr Surg 31:407-410, 1996

27. McLorie GA, Merguerian PA, DeMaria JE, et al: Injuries to the genitourinary system during a posterior sagittal approach and their repair. Br J Urol 81:76, 1998 (Suppl 2)

28. Pacheco BA, Cañas J, Suarez R, et al: Posterior urethral diverticula after imperforate anus repair. BAPS XLV Annual International Congress, Bristol, Poster presentation, 1998 